\title{
Influence of buoyancy on floating zone convection of small Bond number
}

\author{
W. R. HU and Z. M. TANG \\ Institute of Mechanics, Chinese Academy of Sciences, P.O. Box 2554, 15, Zhong Guan Cun Road, \\ Beijing 100080, China
}

(Received for publication 15 December 1993)

\begin{abstract}
Gravity may influence the velocity and temperature distributions, buoyancy may induce Rayleigh instability and the instability may be excited due to the change of free surface shape associating with gravity in the thermocapillary convection. These effects have been studied in the present paper. The results show that gravity may have an important effect in thermocapillary oscillatory convection even for the cases of small Bond number experiments either on the ground or in space.
\end{abstract}

\section{INTRODUCTION}

THE GROUND-BASED experiments of half floating zone are often used to study the thermocapillary effects, which may be dominant in the microgravity environment, by reducing the typical length scale of the liquid bridge to satisfy the condition of small Bond number. Experimental results of transparent mediums have shown that the flow will be changed from symmetric steady convection to asymmetric oscillatory convection logether with the onset of temperature oscillation and free surface vibration if the applied temperature difference is larger than a critical value for either the upper rod heated (URH) case or lower rod heated (LRH) case [1-3]. The mechanism of onset of the oscillation in the floating zone convection has been studied. One opinion considers that the oscillation is excited by thermocapillary instabilities in a simplified model of liquid bridge with infinite length and uniform temperature gradient, such as the Hydrothermal Instability [4] or Surface Wave Instability [5] suggested by Smith and Davis. Another opinion was suggested by Ostrach and Kamotani, who believe that the oscillation does not come due to the instability but due to the free surface deformation, and the deformation at the corner region near the heated rod may induce time lag between mass and heat flux processes, and then induces the oscillation in the liquid bridge [6]. More realistically, the instability analyses by using the numerical result of steady half floating zone convection as the basic state have been completed $[7,8]$. Furthermore, the numerical simulations of evolutionary processes responding to the increasing applied temperature difference were tested $[9,10]$, and these tests may shed light on the transition process from steady to oscillating convection although, sometimes, the mechanism cannot be given clearly.

At the present time, nearly all experiments of half floating zones deal with the transparent fluid medium of large Prandtl number. Most experiments have been completed in the laboratory on the ground in reducing typical scale to satisfy the condition of small Bond number. Microgravity experiments have measured only the temperature distributions in addition to the flow pattern, and lack of space experiment opportunities for larger typical length scale of floating zone gave limited data, which should be repeated and confirmed. However, the measurements of free surface vibration in the ground-based experiments show that, the onset of temperature oscillation always accompanies the free surface wave, which propagates in screwy approach. It seems that these features are different from the theoretical modes of capillary instability such as hydrothermal instability [4].

Recently, the influence of gravity on the thermocapillatory oscillatory convection has been analyzed by the authors, and the effects may be summarized as follows.

- Changing the flow pattern and temperature distribution due to buoyancy.

- Inducing the Rayleigh instability inside the liquid bridge.

- Exciting the oscillation due to the change of free surface shape by gravity.

These factors will change the distributions of velocity and temperature, and, then, may induce the onset of oscillation. Of course, these factors will be coupled with the thermocapillary effects. The influence of gravity will be discussed in detail in the following sections. The results imply that the effect of gravity is not negligible even in the low- and microgravity environment.

\section{INFLUENCE OF BUOYANCY}

At first, discussions are concentrated on the groundbased experiment of half floating zone, which is usually designed in a small length scale for small Bond number. Either case of upper rod heated (URH) or 


\section{NOMENCLATURE}

$A \quad$ aspect ratio

$g$ gravitational acceleration

I typical length

$\operatorname{Pr} \quad$ Prandtl number

$R a_{\mathrm{s}} \quad$ Rayleigh number of space experiment

$R a_{\delta} \quad$ Rayleigh number of ground-based experiment.

Greek symbols

$\beta \quad$ thermal expansion coefficient $\delta q \quad$ microgravity level

d) local typical length

$\delta T$ local typical temperature

difference

$\Delta T$ applied temperature

difference

$(\Delta T)$. critical applied temperature

difference

$\kappa$ thermal diffusion coefficient

$v \quad$ viscosity coefficient. lower rod heated (LRH) is analyzed. Thermocapillary convection will be driven by the non-uniform surface tension due to the applied temperature difference $\Delta T$ between the upper and lower rods, and the buoyancy will drive the convection at the same time and the influence becomes gradually stronger during the increasing $\Delta T$. There is always a inner region of liquid bridge where the temperature gradient reverses direction if the applied temperature difference is larger. Therefore, a buoyancy driven flow will be induced in the region near the symmetric axis for the case of LRH and in the middle upper region for the case of URH as shown in Fig. 1. The temperature and flow field distributions are given, respectively, in Figs. 2 and 3 for four cases of geometric aspect ratio. The results show that the convection cell is pushed toward the upper corner by the buoyancy driven flow in the temperature gradient reversed region.

The temperature gradient reversed region is important not only for buoyancy driven flow, but also for exciting the Rayleigh instability even in the case of URH, which is usually considered as the case of Rayleigh stability and used in the ground-based experiment of small Bond number to simulate the case in microgravity environment. Rayleigh number may be defined as follows for temperature gradient reversed region with typical length scale $\delta l$ and temperature difference $\delta T$ in the opposite direction of gravity

$$
R a_{\delta}=\beta g(\delta T)(\delta l)^{3} / \kappa v
$$

where $\beta, \kappa$ and $v$ are, respectively, the coefficient of thcrmal expansion, thermal diffusion and viscosity. It could be seen that the Rayleigh number $R a_{\delta}$ is $10^{3}$ in order of magnitude for most cases of thermocapillary oscillatory convections of larger Marangoni number in half floating zone with small Bond number, and, in this case, the buoyancy convection may be driven. Furthermore, the Rayleigh instability will be excited if the Rayleigh number $R a_{i}$ is relatively larger, and detailed discussions in order of magnitude were given elsewhere [11].

It should be noted that, the Rayleigh number is proportional to the cubic length scale. The groundbased experiment of small Bond number is usually a few millimeters in length scale: however, the length scale of space experiments is usually at least several centimeters and is larger by one or two orders of magnitude than the ground-hased one. The $R a_{s}$ of

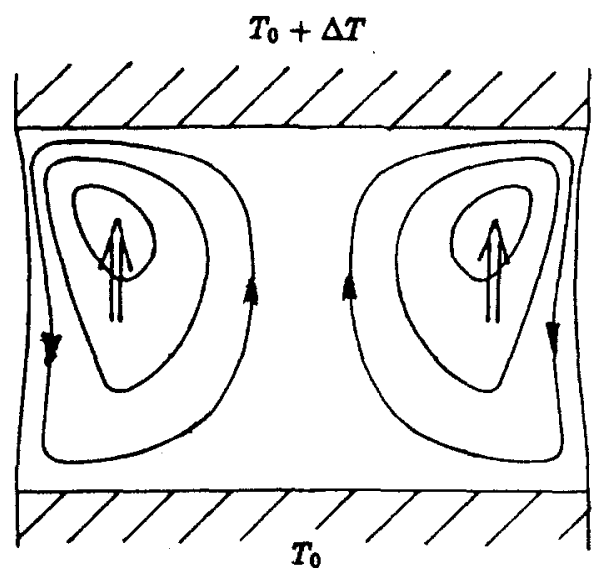

URH

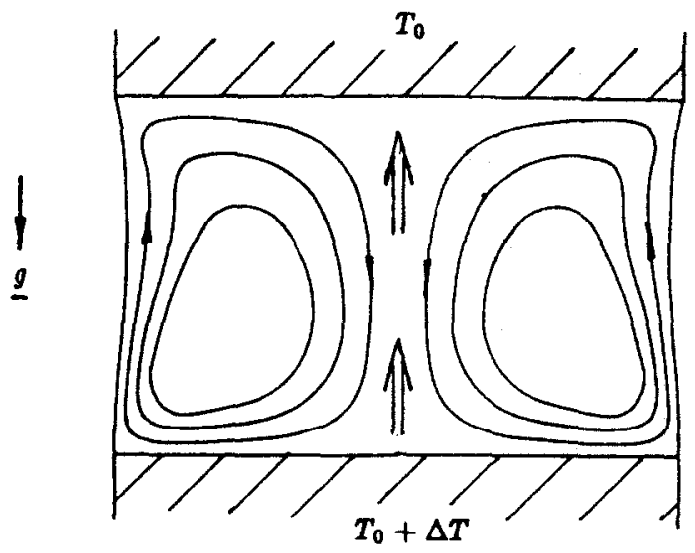

LRH

FIG. 1. The typical convection driven by thermocapillary effect (single arrow) and by buoyancy effect (double array) for the cases of URH (left) and LRH (right) 

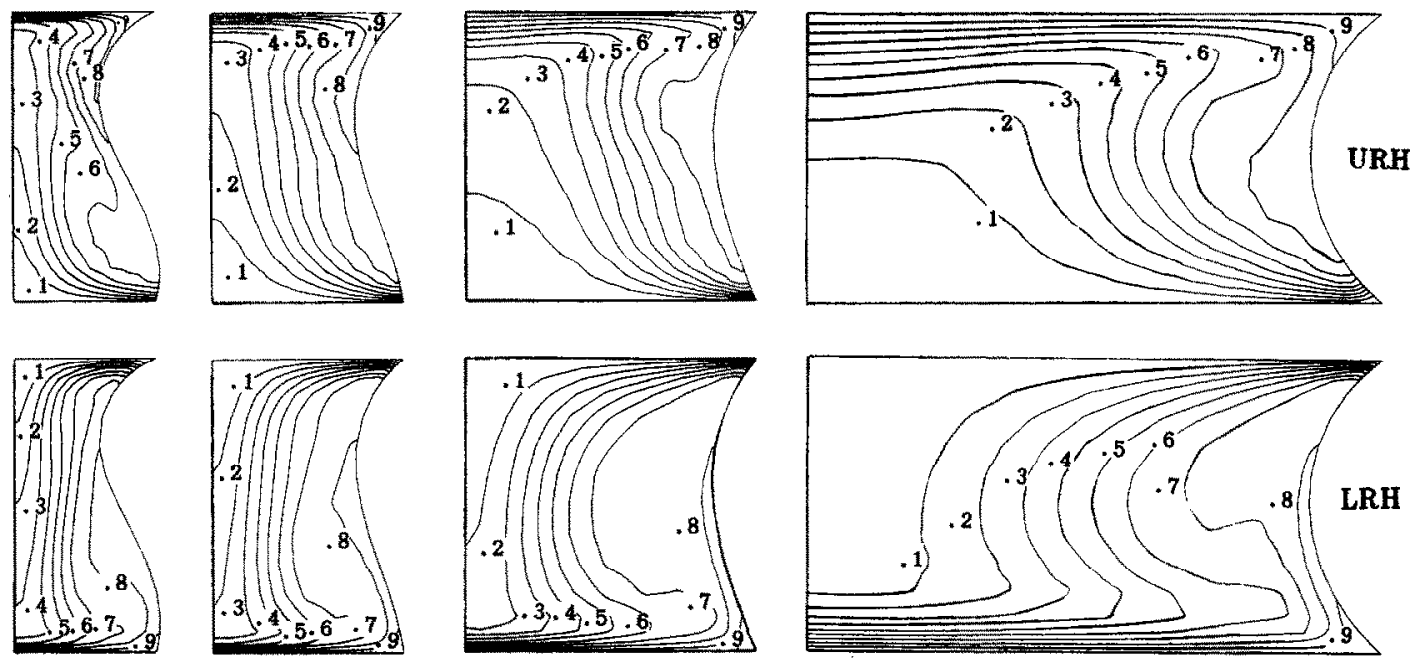

FIG. 2. Temperature profiles in liquid bridge of URH (upper) and LRH (lower) for different aspect ratios, where Marangoni number $M a=10839$ and Prandtl number $\operatorname{Pr}=105.8$.
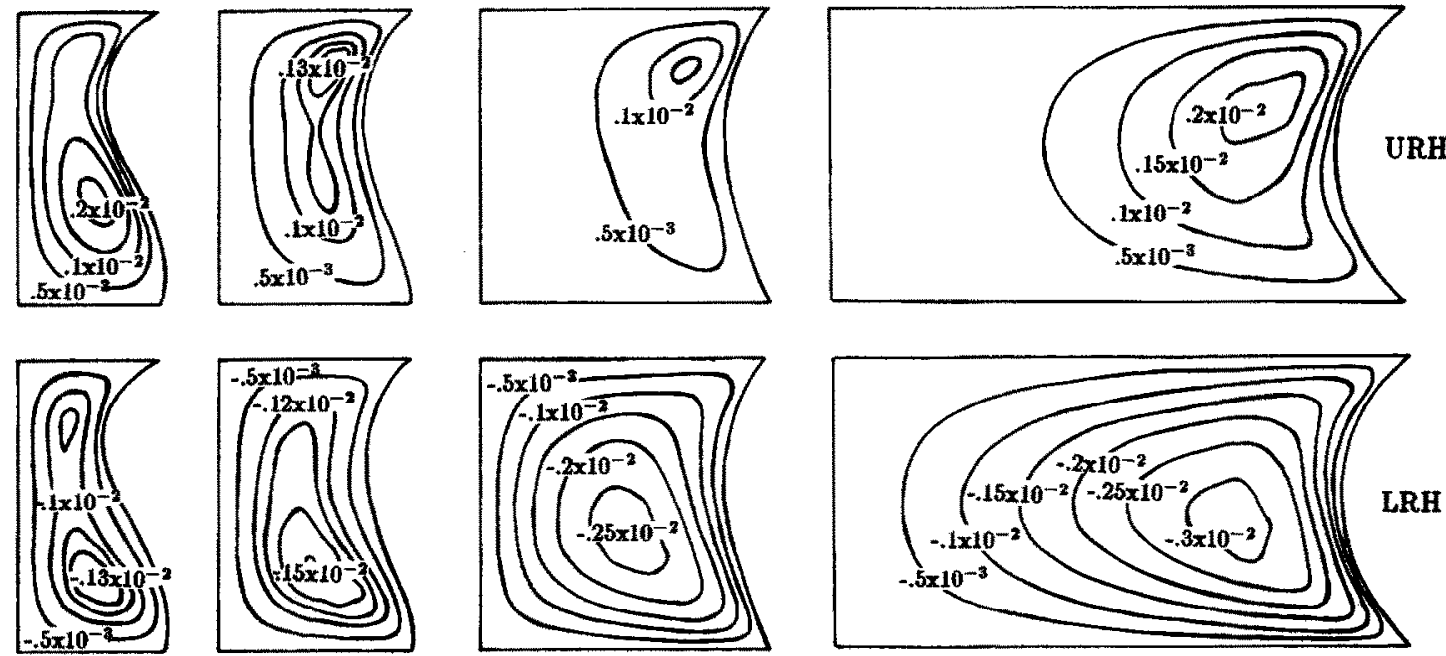

FIG. 3. Velocity distributions in liquid bridge of URH (upper) and LRH (lower) for different aspect ratios, where Marangoni number $M a=10839$ and Prandtl number $\operatorname{Pr}=105.8$.

ground-based experiments may have the same order of magnitude, or even be smaller than the Rayleigh number of space experiments $R a_{\mathrm{s}}$ with typical applied temperature difference $\Delta T$ and length scale $l$ even in the gravity level $\delta g=O\left(10^{-3}-10^{-5} \mathrm{~g}\right)$ environment, that is

$$
\begin{aligned}
R a_{\delta} & =O\left(R a_{s}\right) \\
& =O\left(\beta \delta g \Delta T l^{3} / \kappa v\right) .
\end{aligned}
$$

This implies that the buoyancy convection may be driven and the Rayleigh instability may be excited for space floating zone experiments of larger typical length scale even in the microgravity environment [12].

\section{CRITICAL GRAVITY LEVEL FOR ONSET OF OSCILLATION}

Quantitative analyses show that the gravity may induce the Rayleigh instability in the liquid bridge if the applied temperature difference is larger than a critical value. In this case, there should be a critical gravity level for onset of oscillation. This idea has been tested by the numerical simulation for unsteady and two-dimensional model of liquid bridge floating in the gap of two parallel plans with infinite extension under a gravitational field perpendicular to the planes, and the gravity may be decreased from ground level. A liquid bridge of silicon oil of $4 \mathrm{~mm}$ in height and aspect ratio $A=1$ is analyzed in detail, and the Prandtl number $P r=105.8$ is larger.

The temperature and velocity fields in the liquid bridge were calculated by starting with a small applied temperature difference $\Delta T$, and then, increasing the value of $\Delta T$. The time dependent evolution of the fields was given numerically under fixed physical parameters including gravity level. The convection fields transformed from a smooth distribution into either a state of persistent oscillation or a state tending towards 
smooth distribution after a transient oscillation at a certain range of parameters as shown in Fig. 4. The instability is started by the onset of the persistent oscillation, which gave the associated critical value. Using this procedure, we can test the influence of gravity level on the onset of oscillation, and the results were summarized in Fig. 5 [10]. It could be seen that, the values of critical applied temperature difference $(\Delta T)_{\mathrm{c}}$ in Figs. 4 and 5 are much larger than the values in real cases of half floating zone experiments. These results come from the simplified two-dimensional model, and may shed light on the mechanism studies although the order of magnitude is larger.

According to the numerical test, we obtain again that there is a critical gravity level or a critical Bond number, because Bond number is proportional to the gravity level for fixed aspect of liquid bridge and medium of liquid. The numerical results show that thermocapillary convection in a small scale and upper wall heated liquid bridge may excite oscillation for certain conditions of gravity level and applied temperature difference. The existance of critical gravity level or critical Bond number for fixed geometry and applied temperature difference implies that, the larger the gravity level, the smaller the critical temperature difference, and then, the ease of exciting the oscillation. It means that the temperature gradient in the liquid bridge may turn parallel to the direction of gravity if the applied temperature difference is large enough even for the case of upper heated wall, which is usually believed to be stable. Therefore, the oscil- lation in thermocapillary convection may be excited by buoyancy instability if there is gravity, and the buoyancy may still be an important reason for exciting the oscillation in this flow

\section{INFLUENCE OF GRAVITY VIA FREE SURFACE SHAPE}

The free surface shape is sensitive about the gravity, that is, the shape is symmetric to the central plane in the gravityless environment, and tends to have relatively larger radii along the gravity direction. On the other hand, thermocapillary convection is driven by the non-uniformity of surface tension, and could be considered, in some senses, as the shearing flow with returning circle. The shearing flow may induce the instability, and the shape of free surface associated with the gravity level will influence the onset of oscillation in the liquid bridge of floating zone.

To analyze the detail, four typical free surface shapes associated with the cases on the ground-based gravity $\lg$ (Fig. 6(a)) and in low-gravity environment $10^{-3} g$ with concave shape (Fig. 6(b)), cylinder (Fig. 6(c)) and convex shape (Fig. 6(d)) were considered. Using the unsteady and two-dimensional model of half floating zone, we test the time dependent evolution of the temperature and flow fields for the same physical parameters except the gravity. The results show that in case (a) it is much easier to cause the onset of persistent oscillation than other cases, and
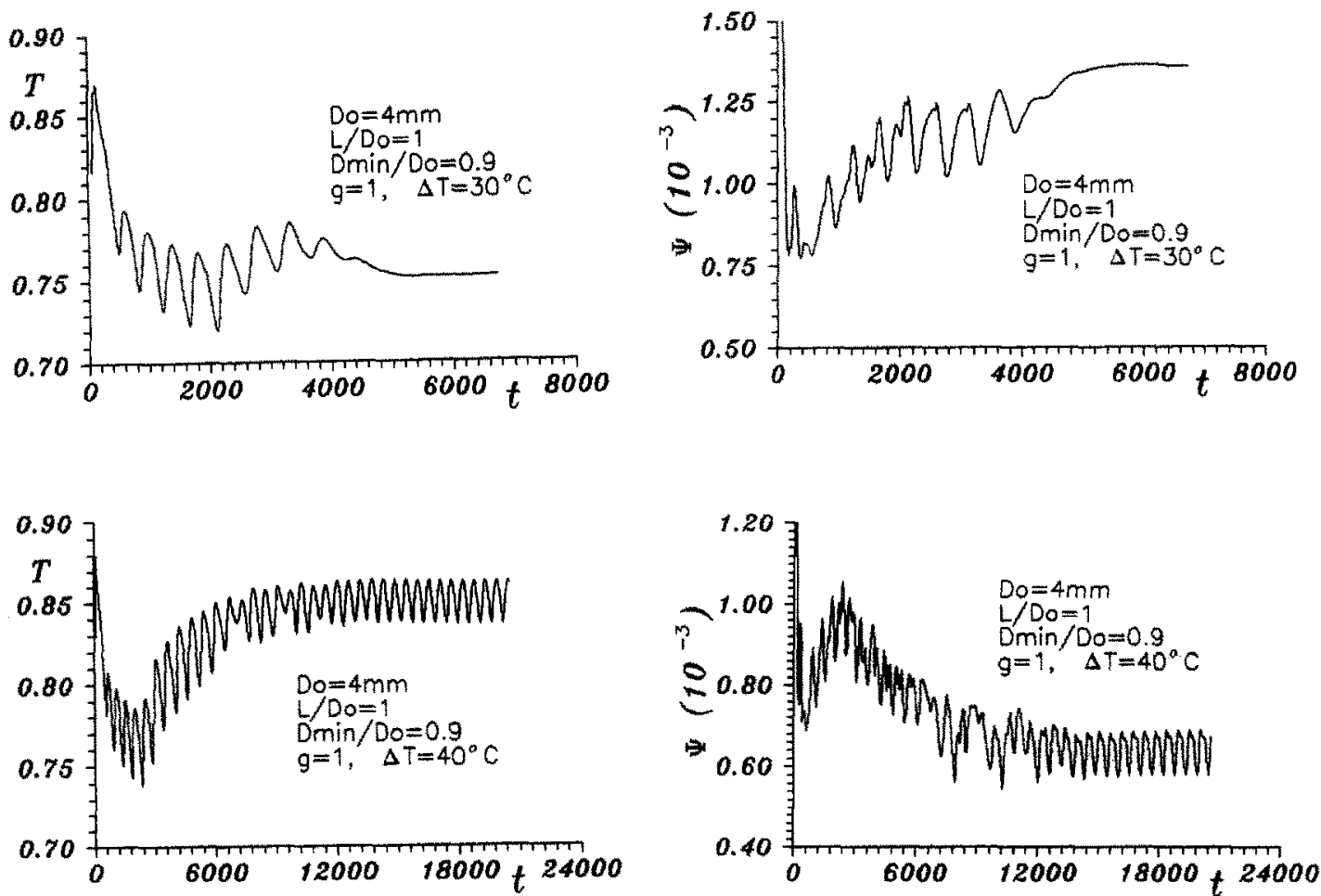

FIG. 4. Transition from smooth fields to transient oscillation (upper) and to persistent oscillation (lower) of temperature field (left) and velocity (right) field. 


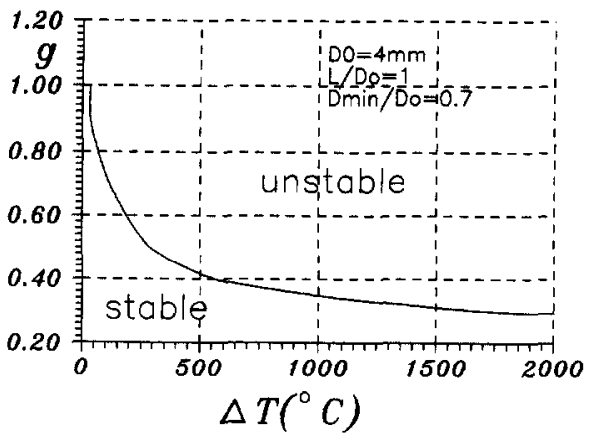

FIr. 5. Marginal stability curve of gravity level depending on Marangoni number.

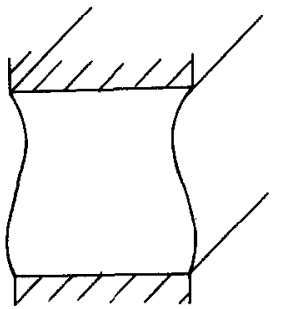

(a) $g=1 g_{0}$

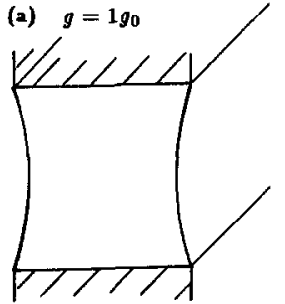

(b) $g=10^{-3} g_{0}$

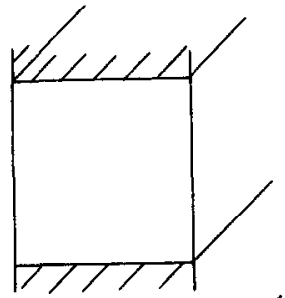

(c) $g=10^{-3} g_{0}$

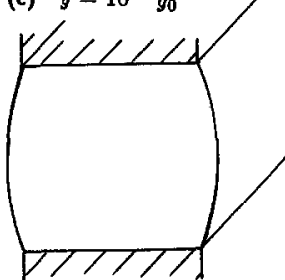

(d) $\mathrm{g}-10^{-3} y_{0}$
FIG. 6. Models of two-dimensional bridges with different free surface shapes under gravity level of $1 \mathrm{~g}$ (a) and $10^{-3} \mathrm{~g}$ (b, c and d).

this oscillatory mechanism seems dependent on the free surface shape associating with gravity [13].

It should be noted that the liquid bridges of four cases have different volumes, and the results may be also explained by the effect of liquid bridge volume. It is proved that the critical condition depends really on the volume of liquid bridge: however, the critical value of applied temperature difference varies in the range less than one time, which is much smaller than the test simulation.

\section{CONCLUSION}

The influence of gravity on the floating zone convection of small Bond number has been studied. Gravity may induce the buoyancy convection, excite the Rayleigh instability and shearing instability due to the configuration of free surface associated with gravity influence. The results show that gravity is one of the sensitive factors for onset of oscillation.

Several mechanisms have been suggested to explain the onset of oscillation in the half floating zone. Until now, none have been proved by experiments. The situation may be more complex than the simplified theoretical models, and also the coupling of two or more mechanisms may operate at the onset of oscillation.

One of the important problems is the growth rate of the oscillatory modes. The onset of oscillation may be excited by several modes, and the dominant mode is the one of fast growth rate. However, the studies have little idea on the growth rate at the present time, and the onset of oscillatory convection in the half floating zone is still a open problem to be studied.

\section{REFERENCES}

1. Z. H. Cao, H. T. You, Z. M. Tang and W. R. Hu, Experimental investigation of thermocapillary convection in half floating zone, Adv. Space Res. 11 (7), 229-232 (1991).

2. W. R. Hu, II. T. You and Z. H. Cao, Free surface oscillation of thermocapillary convection in liquid bridge of half floating zone, Sci. China A 35, 1101-1 108 (1992).

3. J. 7. Shu, Y. I. Yao and W. R. Hu, Free surface vibration in oscillatory convection of half floating zone, Sci. China A 36, 326-332 (1993).

4. M. K. Smith and S. H. Davis, Instability of dynamic thermocapillary liquid bridge, I. Convective instability, J. Fluid Mech. 132, 119-144 (1983).

5. M. K. Smith and S. H. Davis, Instability of dynamic thermocapillary liquid bridge, II. Surface-wave instability, J. Fluid Mech. 132, 145-162 (1983).

6. S. Ostrach, C. Kamotoni and L. Lai, Oscillatory thermocapillary flows, Physico-Chemical Hydrodyn. 6, 585599 (1985).

7. Y. Shen, G. P. Neitzel, D. F. Jankowski and H. D. Mittelmann, Energy stability of thermocapillary convection in a model of the floating-zone crystal-growth process, J. Fluid Mech. 217, 639-660 (1990).

8. H. C. Kuhlmann and H. J. Rath, Hydrodynamic instability in cylindrical thermocapillary liquid bridge, $J$. Fluid Mech. 247, 247-274 (1993).

9. N. D. Kazarinoff and J. S. Wilkowski, Bifurcation of numerically simulated thermocapillary flow in axially symmetric floating zones, Phys. Fluid A2, 1797-1807 (1990).

10. Z. M. Tang and W. R. Hu, Critical Bond number in two-dimensional thermocapillary oscillatory convection, Chinese Sci. Bull. 38, 1249-1252 (1992).

11. W. R. Hu, The influence of buoyancy on the oscillatory thermocapillary convection with small Bond number, Acta Astronautica 18, 593-597 (1990)

12. W. R. Hu and Z. M. Tang, Exciting mechanism of thermocapillary oscillatory convection, Sci. China 34, 1354 1364 (1091).

13. Z. M. Tang and W. R. Hu, Numerical simulation of thermocapillary oscillatory convection, Adri. Space Res 13(7), 183-186 (1993). 